\title{
Amélioration de la résistance des lactocoques aux bactériophages
}

\author{
E Bidnenko 1, R Valyasevi 1, PJ Cluzel 1*, R Parreira 1, \\ A Hillier $1^{\star \star}$, M Gautier 2, J Anba 1, SD Ehrlich 1, MC Chopin 1 \\ ${ }^{1}$ Laboratoire de génétique microbienne, INRA, 78352 Jouy-en-Josas; \\ ${ }^{2}$ Laboratoire de recherches de technologie laitière, INRA, 35032 Rennes, France
}

\begin{abstract}
Résumé - Les phages sont reconnus depuis longtemps comme la principale cause de ralentissement de la croissance des lactocoques composant les levains de fromagerie. La sélection de souches naturelles ou mutantes particulièrement résistantes aux phages ne permettant pas un contrôle optimum des fabrications, une nouvelle approche a été envisagée après analyse des mécanismes de résistance aux phages naturellement présents dans les souches. II s'agit de construire des souches plus résistantes aux phages par accumulation de plusieurs mécanismes de résistance dont les effets s'additionnent. Une telle approche nécessite l'isolement et la caractérisation de mécanismes variés de résistance aux phages. Nous résumons ici le clonage et la caractérisation des gènes codant pour 3 mécanismes d'infection abortive. Les cibles de ces 3 mécanismes ont été localisées sur l'ADN d'un phage. Deux d'entre elles ont été clonées et sont en cours de caractérisation. L'étude de l'interaction entre les gènes codant pour ces mécanismes de résistance et leur cible sur le phage devrait nous apporter les connaissances nécessaires pour un choix rationnel des mécanismes à utiliser.
\end{abstract}

\section{Lactococcus / bactériophage / résistance / infection abortive}

Summary - Improvement of phage resistance in Lactococcus. The need to improve phage resistance in lactococcal strains used for cheesemaking has long been recognised. Recent molecular and genetic studies of these microorganisms have given insight into phage defense mechanisms and opened new possibilities for the improvement of lactococcal phage resistance. Naturally occurring lactococci with a high degree of phage resistance have been shown to possess several types of phage resistance mechanisms including adsorption interference, restriction and modification, and abortive infection. It has been suggested that cheesemaking strains might be improved by introducing several defense mechanisms, acting on a broad range of phages at different steps of their life cycle, into these strains. Such an approach relies on the availability of a number of highly efficient, well-characterized phage resistance mechanisms and on the development of efficient food grade transfer procedures in industrial strains. In this paper, we consider only the characterization of phage abortive infection mechanisms (Abi). In the presence of an Abi mechanism, the efficiency of phage adsorption remains the same but phages multiply only in a fraction of the infected cells. In addition, burst size is decreased. As a result, phage number does not increase and the culture grows normal-

\footnotetext{
- Pierre-Jean Cluzel, qui a mené une partie importante des travaux décrits, est décédé prématurément le 27 mars 1991. "** Adresse permanente : CSIRO, Dairy Research Laboratory, PO Box 20, Highett, Victoria 3190, Australie.
} 
ly. The Abi mechanisms are therefore of particular interest for the improvement of lactococcal phage resistance.

In order to obtain more insight in the mode(s) of action of Abi mechanisms, we cloned 3 different Abiencoding DNA fragments. These 3 determinants were compared to thcse already published or currently under characterization in other laboratories. Among 7 genes compared, 6 are different including the 3 we have cloned. These results indicate that Abi mechanisms are diverse and should have different modes of action. Two of the cloned determinants were subcloned and sequenced. In both cases, an iso-ISS1 element was present upstream of the gene coding for the Abi+ phenotype. The expression of both abi genes was directed by a promoter present in this IS element. Therefore, in addition to a contribution to lactococcal gene mobility, ISS1 is able to participate in gene expression.

In order to understand how the abi gene products interfere with phage development, we have compared the small isometric-headed phage blL66 to one of its spontaneous mutants able to overcome abi105. The mutation was localised on a 3-kb DNA fragment from the phage. In presence of abi-105, the $3 \mathrm{~kb}$ fragment carrying the mutation allows multiplication of several unrelated phages sensitive to abi105. The gene involved in the multiplication of unrelated phages was localized on the 3-kb DNA fragment and sequenced. The protein it specifies shares no homology with proteins from the data banks. Studies are currently in progress to determine how the abi gene product interferes with the phage genes.

Lactococcus / bacteriophage / resistance / abortive infection

\section{INTRODUCTION}

Les phages sont reconnus depuis longtemps comme la principale cause de ralentissement de la croissance des bactéries composant les levains de fromagerie. Les conséquences de tels ralentissements, au niveau de l'organisation du travail et de la qualité des produits, varient selon les types de fabrication et sont particulièrement perceptibles dans celles utilisant des lactocoques.

Des procédés technologiques ont été développés de façon a limiter l'infection des levains par les phages (milieux de culture spéciaux, suspensions concentrées congelées ou lyophilisées). II est néanmoins difficile d'éviter toute infection des levains au cours des fabrications. Des efforts ont donc été faits pour sélectionner des souches de lactocoques particulièrement résistantes aux phages. Les 2 approches utilisées, (recherche de souches naturelles et sélection de mutants), sont cependant limitées et ne permettent pas de maîtriser le problème de façon satisfaisante. Moins de $1 \%$ des souches sauvages isolées ont à la fois une forte résistance aux phages et les propriétés requises pour leur production industrielle et pour leur utilisation technologique. Leur durée d'utilisation industrielle est très variable. Les mutants résistants aux phages qui peuvent être aisément obtenus pour certaines souches, perdent parfois les caractéristiques technologiques de la souche d'origine et de nouveaux phages capables de les attaquer apparaissent plus ou moins rapidement lors de leur emploi en fabrications industrielles (Gasson et Davies, 1984).

L'utilisation des techniques génétiques a permis de comprendre pourquoi certaines souches sont plus résistantes aux phages que d'autres, et de proposer une nouvelle approche pour renforcer la résistance des souches aux phages. Plusieurs souches de lactocoques présentant une résistance élevée aux phages ont été étudiées dans différents laboratoires. Toutes ces souches avaient accumulé plusieurs 
types de mécanismes : blocage de l'adsorption, système de restriction/modification ou mécanisme d'infection abortive (pour des revues, voir : Daly et Fitzgerald, 1987; Klaenhammer, 1987; Sanders, 1988). L'existence de souches particulièrement résistantes suggère qu'il doit être possible d'améliorer d'autres souches en y accumulant des mécanismes variés de résistance aux phages. En effet, si chaque mécanisme pris indépendamment peut être contourné par un phage, la probabilité que ce phage parvienne à surmonter plusieurs mécanismes différents est beaucoup plus faible. Dans un premier temps, en l'absence de réglementation sur l'utilisation de souches recombinantes en agroalimentaire, on peut mimer la nature de façon dirigée et introduire des plasmides conjugatifs dans des souches aux aptitudes technologiques particulièrement intéressantes. Cette approche a déjà été utilisée (Sanders et al, 1986). Ces possibilités sont cependant limitées par l'aptitude des plasmides à conjuguer d'une souche à une autre, la compatibilité et la stabilité des plasmides transférés dans une même souche. Une approche plus souple et certainement beaucoup plus performante consistera dans un deuxième temps à utiliser les techniques du génie génétique. Cela permettra d'utiliser une gamme plus large de mécanismes de résistance en employant les gènes chromosomiques qui, dans la nature, ne sont pas facilement transférés d'une souche à une autre, ou en développant d'autres systèmes tels que ceux basés sur les ARN antisens (Kim et Batt, 1991; Chung et al, 1992). II sera également possible de surexprimer ces mécanismes, de les stabiliser en les insérant dans le chromosome, et de les accumuler dans des souches choisies pour leurs propriétés technologiques.

Une telle approche nécessite l'isolement et la caractérisation de plusieurs mécanismes de résistance aux phages, l'éluci- dation du mode de fonctionnement de ces mécanismes et le développement de systèmes de transfert de gènes à la fois efficaces dans les souches industrielles et acceptables par les consommateurs et les organismes réglementaires. Bien que travaillant sur ces 3 points, nous aborderons seulement ici l'étude du fonctionnement des mécanismes d'infection abortive (Abi).

\section{ÉTAT DES CONNAISSANCES SUR LES MÉCANISMES D'INFECTION ABORTIVE}

Parmi les mécanismes de résistance aux phages identifiés jusqu'à présent, les Abi nous semblent les plus prometteurs. En effet, en présence d'un mécanisme $\mathrm{Abi}$, les phages s'adsorbent normalement sur la bactérie mais seule une fraction des cellules infectées permet la multiplication des phages $(0,04$ à $38 \%$ selon I'Abi) et cela avec un rendement unitaire diminué (fig 1 ). La multiplication des phages avorte donc dans la majorité des cas. Les phages libérés ne peuvent à leur tour se multiplier que dans un faible pourcentage des cellules infectées. Dans ces conditions, le nombre

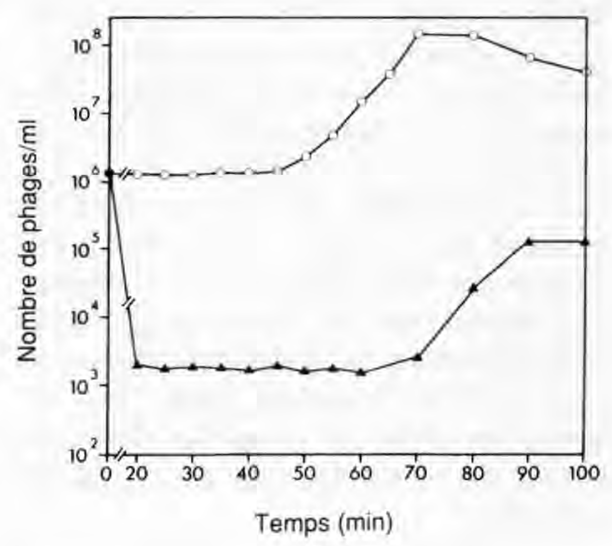

Fig 1. Courbe de croissance du phage blL66 sur les souches L lactis subsp lactis IL1403 (O) et IL403 (abi-420) (A). 
de phages n'augmente pas, voire diminue, et ne gène pas la croissance bactérienne. Ces mécanismes fonctionnent donc comme de véritables pièges à phages et semblent particulièrement utiles pour l'amélioration de la résistance aux phages des souches industrielles.

Plusieurs mécanismes $\mathrm{Abi}$ ont été décrits chez les lactocoques. Le plus étudié de ces mécanismes, codé par le plasmide conjugatif pTR2030, a été mis en évidence à l'origine dans une souche de $L$ lactis subsp lactis (Klaenhammer et Sanozki, 1985). Transféré dans plusieurs souches de $L$ lactis subsp lactis et de $L$ lactis subsp cremoris ce plasmide a fortement inhibé la multiplication de tous les petits phages isométriques étudiés (Jarvis et Klaenhammer, 1986; Sing et Klaenhammer, 1986). En revanche, les phages à tête allongée et les gros phages isométriques étaient insensibles à l'action de pTR2030 ou subissaient seulement une faible diminution de leur rendement unitaire (Jarvis et Klaenhammer, 1986). Une telle corrélation entre groupes morphologiques de phages et sensibilité aux mécanismes de multiplication abortive a été observée pour d'autres plasmides (Daly et Fitzgerald, 1987; Froseth et al, 1988; Murphy et al, 1988; Steele et McKay, 1989) qui sont en général plus actifs sur les phages à tête isométrique que sur ceux à tête allongée. Deux exceptions ont cependant été décrites : les plasmides pIL105 (Gautier et Chopin, 1987) et pAJ1106 (Jarvis et al, 1989) qui agissent avec la même efficacité sur des phages de type isométrique et allongé. Les mécanismes de résistance codés par ces 2 plasmides se distinguent également par le fait qu'ils sont les seuls pour lesquels l'apparition de phages mutants capables de surmonter leur action ait été rapportée.

Trois gènes codant pour un phénotype Abi ont été clonés et séquences à ce jour (Hill et al, 1990; Coffey et al, 1991; Cluzel et al, 1991). Deux d'entre eux sont identiques. L'absence d'homologie entre les protéines codées par les gènes séquences et les protéines des banques de données empêche d'émettre toute hypothèse quant à leur(s) mode(s) d'action. Des connaissances dans ce domaine sont cependant indispensables pour choisir rationnellement les mécanismes accumulables dans une même souche et étudier les possibilités d'accroìtre leur efficacité en modifiant l'expression des gènes qui les codent.

\section{CARACTÉRISATION DE TROIS MÉCANISMES D'INFECTION ABORTIVE}

Afin de contribuer à la caractérisation des mécanismes de type Abi, nous avons cloné à partir de l'ADN total de 2 souches de lactocoques et du plasmide plL105 (Gautier et Chopin, 1987), 3 fragments d'ADN conférant le phénotype Abit à la souche sans plasmide $L$ lactis subsp lactis IL1403 (Chopin et al, 1984). Le clonage a été réalisé avec le vecteur plL253 (Simon et Chopin, 1988) directement dans IL1403 en sélectionnant les transformants résistant à l'érythromycine et au phage blL66 (Cluzel et al, 1991). Les 3 fragments d'ADN codent pour un mécanisme ayant un spectre d'activité différent sur une série de phages choisis comme modèles (tableau I). Des comparaisons de séquence et des hybridations d'ADN de ces 3 fragments avec les gènes abi provenant des plasmides pTR2030 (Hill et al, 1990) et pCl829 (Coffey et al, 1991) ainsi qu'avec d'autres gènes en cours de caractérisation (Klaenhammer, communication personnelle; Fitzgerald, communication personnelle), nous ont permis d'établir que les 3 gènes caractérisés dans notre laboratoire sont différents des autres et que sur les 7 gènes étudiés, 2 seulement sont identiques. Ces résultats indiquent une diversité 
Tableau I. Spectre d'action des systèmes $a b i-105, a b i-416$ et $a b i-420$ sur différents phages de lactocoques.

\begin{tabular}{|c|c|c|c|c|c|}
\hline & \multicolumn{3}{|c|}{ Phages isométriques } & \multicolumn{2}{|c|}{ Phages prolates } \\
\hline & bIL41 & bIL66 & blL170 & bIL67 & bIL188 \\
\hline IL1043 & $+a$ & + & + & + & + \\
\hline IL1403 (abi-420) & $-b$ & - & + & + & + \\
\hline IL1403 (abi-416) & + & - & - & + & + \\
\hline IL1403 (abi-105) & - & - & - & - & - \\
\hline
\end{tabular}

${ }^{a}$ Croissance normale du phage; ${ }^{b}$ inhibition de la croissance du phage.

assez grande des Abi et peut-être diffèrents modes d'action. Ils sont en accord avec le fait que l'accumulation de plusieurs Abi dans une souche entraîne une addition de leurs efficacités comme si les quelques phages échappant à un premier mécanisme étaient inhibés par le second, et ainsi de suite (Daly et Fitzgerald, 1987; MC Chopin, résultats non publiés).

Après sous-clonage, 2 des fragments ont été séquencés. L'organisation de ces fragments est schématisée sur la figure 2 . Dans les 2 cas, une séquence d'insertion se trouve en amont du gène codant pour le phénotype Abit. Cet élément d'insertion présente $99,5 \%$ d'homologie au niveau nucléotidique avec celui (ISS1) identifié pour la première fois au voisinage des gènes lactose (Polzin et Shimizu-Kadota, 1987). Des séquences d'insertion de ce type avec une conservation de 79 à $99,5 \%$ au niveau nucléotidique ont également été trouvées au voisinage des gènes protéase (Haandrickman et al, 1990) et des gènes de résistance aux phages (Romero et Klaenhammer, 1990). ISS1 est en fait très fréquente chez les lactocoques, où elle est présente sur les plasmides et le chromo-
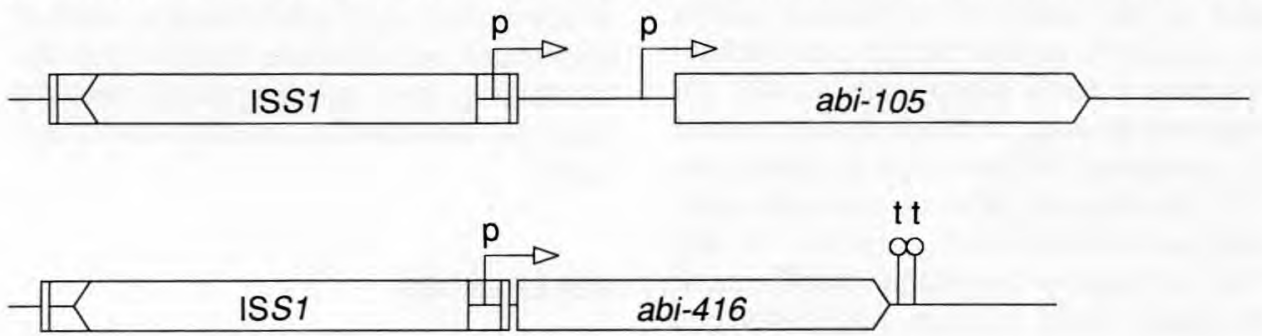

$0.5 \mathrm{~kb}$

Fig 2. Organisation des fragments d'ADN portant les gènes $a b i-105$ et abi-416. $p$ : promoteur; $t$ : terminateur. 
some de la plupart des souches en une ou plusieurs copies. Nous avons établi que ISS1 apporte un promoteur indispensable à l'expression du gène abi-416 (Cluzel et $a l, 1991)$. Le gène abi-105 s'exprime à partir du promoteur situé en amont. Cependant, le promoteur apporté par ISS1 augmente son niveau d'expression et l'efficacité du mécanisme de résistance pour lequel il code. ISS1 peut par conséquent, non seulement jouer un rôle dans la mobilité des gènes de lactocoques, comme suggéré par Gasson (1990), mais aussi dans leur expression.

Les gènes abi-416 et $a b i-105$ identifiés par la perte du phénotype $\mathrm{Abi}+$ qui accompagne leur délétion, cocient pour des protéines ne présentant aucune homologie avec celles des banques de données.

La deuxième étape dans l'élucidation du fonctionnement des Abi est de comprendre comment le produit des différents gènes $a b i$ interfère avec la multiplication des phages. Pour cela, nous avons commencé par comparer le phage bIL66 à un phage mutant résistant à $a b i-105$. Des phages bIL66 mutants apparaissent spontanément à fréquence élevée (10-4) (Gautier et Chopin, 1987). Six des 7 fragments EcoRI de I'ADN d'un de ces phages mutants d'une part et du phage blL66 d'autre part ont été clonés dans Bacillus subtilis en utilisant le vecteur pIL253 puis transférés dans $L$ lactis subsp lactis IL1403. Un fragment de $3 \mathrm{~kb}$ du phage mutant permet la croissance, en présence du gène $a b i$ 105 , du phage bIL66 et de 3 phages hétérologues normalement sensibles à $a b i$ 105. Le fragment homologue cloné à partir du phage blL66 sauvage ne permet pas une telle croissance. La mutation qui confère ce phénotype est donc portée par le fragment de $3 \mathrm{~kb}$. Nous avons établi génétiquement que ce fragment code pour une molécule diffusible permettant la croissance des phages hétérologues par complémentation. Par sous-clonage, séquen- çage et tests de complémentation, nous avons pu identifier un gène dont le produit est responsable de cette complémentation. La protéine codée par ce gène n'a pas d'homologie avec celles des banques de données. Nous ne pouvons donc pas, pour l'instant, émettre d'hypothèse sur la fonction possible de cette protéine.

\section{PERSPECTIVES}

L'étude de l'interaction entre le produit du gène $a b i-105$ et son gène cible sur le phage blL66 devrait nous permettre de progresser dans la compréhension du fonctionnement moléculaire de ce mécanisme de résistance. Cela, joint aux études menées parallèlement sur $a b i-416$ et $a b i-420$ et à celles réalisées dans d'autres laboratoires, devrait nous apporter une connaissance globale sur les mécanismes de type Abi. II sera alors possible de choisir rationnellement les mécanismes à accumuler pour bloquer les différentes étapes de la multiplication intracellulaire des principaux types de phages. Une même série de mécanismes de résistance pourrait être introduite dans différentes souches préalablement ameliorées pour d'autres caractères d'intérêt technologique. Ainsi, dans les années à venir, des souches répondant à des besoins variés et permettant une meilleure maïtrise des fermentations, donc une plus grande régularité dans les fabrications, pourront être construites.

\section{RÉFÉRENCES}

Chopin A, Chopin MC, Moillo-Batt A, Langella P (1984) Two plasmid-determined restriction and modification systems in Streptococcus lactis. Plasmid 11, 260-263

Chung DK, Chung SK, Batt CA (1992) Antisense RNA directed against the major capsid protein of $L$ lactis subsp cremoris bacterio- 
phage 4-1 confers partial resistance to the host. Appl Microbiol Biotechnol 37, 79-83

Cluzel P J, Chopin A, Ehrlich SD, Chopin MC (1991) Phage abortive infection mechanism from Lactococcus lactis subsp lactis, expression of which is mediated by an iso-ISS1 element. Appl Environ Microbiol 57, 3547-3551

Coffey A, Fitzgerald G, Daly C (1991) Cloning and characterization of the determinant for abortive infection of bacteriophages from lactococcal plasmid pCl829. J Gen Microbiol $137,1355-1362$

Daly C, Fitzgerald G (1987) Mechanisms of bacteriophage insensitivity in the lactis streptococci. In: Streptococcal Genetics (Ferretti J, Curtiss R III, eds), American Society for Microbiology, Washington DC

Froseth BR, Harlander SK, McKay LL (1988) Plasmid-mediated reduced phage sensitivity in Streptococcus lactis KR. J Dairy Sci 71, 275-284

Gasson M J, Davies FL (1984) Bacteriophages of dairy lactic-acid bacteria. In: Advances in the Microbiology and Biochemistry of Cheese and Fermented Mik ( Davies FL, Law BA, eds), Elsevier Applied Science Publishers, London

Gasson MJ (1990) In vivo genetic systems in lactic acid bacteria. FEMS Microbiol Rev 87 , 43-60

Gautier M, Chopin MC (1987) Plasmid determined systems for restriction and modification activity and abortive infection in Streptococcus cremoris. Appl Environ Microbiol 53, 923-927

Haandrikmann AJ, Van Leeuwen C, Kok J, Vos $P$, de Vos WM, Venema G (1990) Insertion elements on lactococcal proteinase plasmids. App/ Environ Microbiol 56, 1890-1896

Hill C, Miller LA, Klaenhammer TR (1990) Nucleotide sequence and distribution of the pTR2030 resistance determinant (hsp) which aborts bacteriophage infection in lactococci. Appl Environ Microbiol 56, 2255-2258.

Jarvis AW, Klaenhammer TR (1986) Bacteriophage resistance conferred on lactic streptococci by the conjugative plasmid pTR2030: effects on small isometric-, large isometric-, and prolate-headed phages. Appl Environ Microbiol 51, 1272-1277

Jarvis AW, Heap HA, Limsowtin GKY (1989) Resistance against industrial bacteriophages conferred on lactococci by plasmid pAJ1106 and related plasmids. Appl Environ Microbiol $55,1537-1543$

Kim SG, Batt CA (1991) Antisense mRNAmediated bacteriophage resistance in $L$ lactis subsp lactis. Appl Environ Microbiol 57, 1109-1113

Klaenhammer TR (1987) Plasmid-directed mechanisms for bacteriophage defense in lactic streptococci. FEMS Microbiol Rev 46, 313-325

Klaenhammer TR, Sanozky RB (1985) Conjugal transfer from Streptococcus lactis ME2 of plasmids encoding phage resistance, nisin resistance and lactose fermenting ability: evidence for a high-conjugative plasmid responsible for abortive infection of virulent bacteriophage. J Gen Microbiol 131, 1531-1541

Murphy MC, Steele SL, Daly C, McKay LL (1988) Concomitant conjugal transfer of reduced-bacteriophage sensitivity mechanisms with lactose- and sucrose-fermenting ability in lactic streptococci. Appl Environ Microbiol $54,1951-1956$

Polzin KM, Shimizu-Kadota M (1987) Identification of a new insertion element, similar to gram-negative IS26, on the lactose plasmid of Streptococcus lactis ML3. J Bacteriol 169, 5481-5488

Romero DA, Klaenhammer TR (1990) Characterization of insertion sequence IS946, an IsoISS1 element, isolated from the conjugative plasmid pTR2030. J Bacteriol 172, 4151-4160

Sanders ME (1988) Phage resistance in lactic acid bacteria. Biochimie 70, 411-421

Sanders ME, Leonhard PJ, Sing WD, Klaenhammer TR (1986) Conjugal strategy for construction of fast acid-producing, bacteriophage resistant lactic streptococci for use in dairy fermentations. Appl Environ Microbiol 52, 1001-1007

Simon D, Chopin A (1988) Construction of a vector plasmid family and its use for molecular cloning in Streptococcus lactis. Biochimie 70, 559-566

Sing WD, Klaenhammer TR (1986) Conjugal transfer of bacteriophage resistance determinants on pTR2030 into Streptococcus cremoris strains. Appl Environ Microbiol 51, 1264-1271

Steele JL, McKay LL (1989) Conjugal transfer of genetic material by Lactococcus lactis subsp lactis 11007. Plasmid 22, 32-43 